\title{
The Classification of Pavement Crack Image Based on Beamlet Algorithm
}

\author{
Aiguo Ouyang ${ }^{*}$, Qin Dong, Yaping Wang, and Yande Liu \\ Institute of Optics-Mechanics-Electronics Technology and Application (OMETA), \\ School of Mechanical and Electronic Engineering, East China Jiaotong University, \\ Nanchang 330013, P.R. China \\ ouyang1968711@163.com
}

\begin{abstract}
Pavement distress, the various defects such as holes and cracks, represent a significant engineering and economic concern. This paper based on Beamlet algorithm using MATLAB software to process the pavement crack images and classify the different cracks into four types: horizontal, vertical, alligator, and block types. Experiment results show that the proposed method can effectively detect and classify of the pavement cracks with a high success rate, in which transverse crack and longitudinal crack detection rate reach to $100 \%$, and alligator crack and block crack reach more than $85 \%$.
\end{abstract}

Keywords: Pavement Crack, Beamlet Algorithm, Classification, Transform.

\section{Introduction}

Pavement distress, the various defects such as cracks illustrated in Fig.1, represent a significant engineering and economic concern. Pavement crack image classification is important in an automated pavement inspection system, because it can provide critical information for pavement maintenance.

There has been a significant amount of research during the last two decades in developing image processing algorithm for pavement crack inspection. Chou et al [1]. approached the problem of pavement crack classification by using moment invariant and neural networks. After preprocessing and thresholding into binary images, they calculated $\mathrm{Hu}$, Bamieh, and Zemike moments. Teomete et al [2]. proposed histogram projection to identify cracks within a cropped image. While focused on the severity of cracks, crack classification, was not performed. Moreover, the system cannot detect multiple cracks within an image.In a paper by Bray [3], cracks is performed using a neural network while classification is performed by another neural network. The proposed algorithm has not been tested on real images. Cheng et al [4]. described a neural network based thresholding method to segment and classify pavement images that can be implemented in real time. Tsai et al [5]. presented a critical assessment of various segmentation algorithms for pavement distress detection and classification.

\footnotetext{
* Corresponding author.

D. Li and Y. Chen (Eds.): CCTA 2013, Part II, IFIP AICT 420, pp. 129-137, 2014.

(C) IFIP International Federation for Information Processing 2014
} 


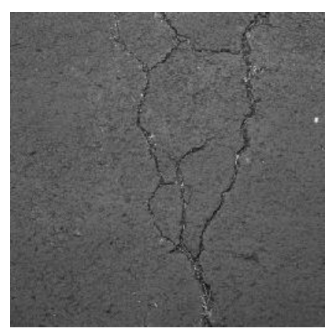

Alligator

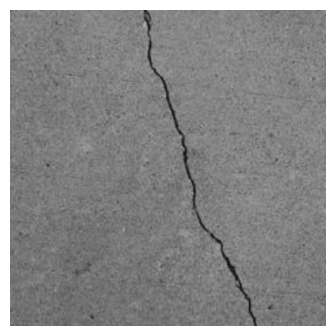

Longitudinal

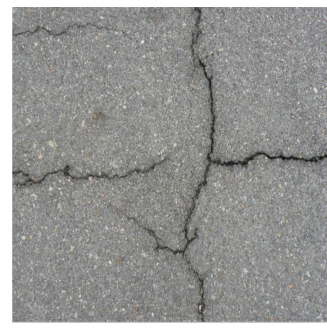

Block

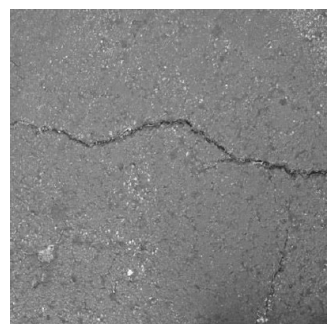

Transverse

Fig. 1. Types of pavement cracks

\section{Beamlet Alogrithm}

\subsection{Beamlet Dictionary}

The beamlet transform is performed in the dynamically partitioned squares of an image. Images are viewed as the continuum square $[0,1]^{2}$ and the pixels as an array of $1 / \mathrm{n}$ by $1 / \mathrm{n}$ squares arranged in a grid in $[0,1]^{2}$. The collection of beamlets is a multiscale collection of line segments occurring at a full range of orientations, positions, and scales[6], as illustrated in Fig.2.

\subsection{Beamlet Transform}

The beamlet transform is defined as the collection of line integrals along the set of all beamlets. Let $f\left(x_{1}, x_{2}\right)$ be a continuous function on 2-D space, where $x_{1}$ and $x_{2}$ are coordinates. The beamlet transform $T_{f}$ of function $\mathrm{f}$ is defined as follows:

$$
T_{f}(b)=\int_{b} f(x(l)) d l, \quad b \in B_{E}
$$

Where $B_{E}$ is the collection of all beamlets. 

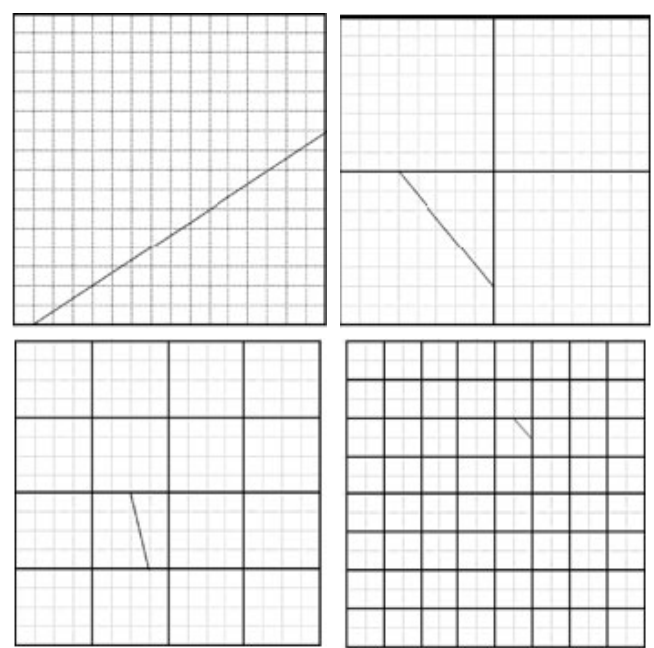

Fig. 2. Four beamlets, at various scales, locations, and orientations

For a digital image, the beamlet transform is a measure of the line integral in the discrete domain. As Fig.3 shows, the beamlet transform for all the points along the beamlet $\mathrm{b}$ is defined as,

$$
f\left(x_{1}, x_{2}\right)=\sum_{i_{1}, i_{2}} f_{i_{1}, i_{2}} \Phi_{i_{1}, i_{2}}\left(x_{1}, x_{2}\right)
$$

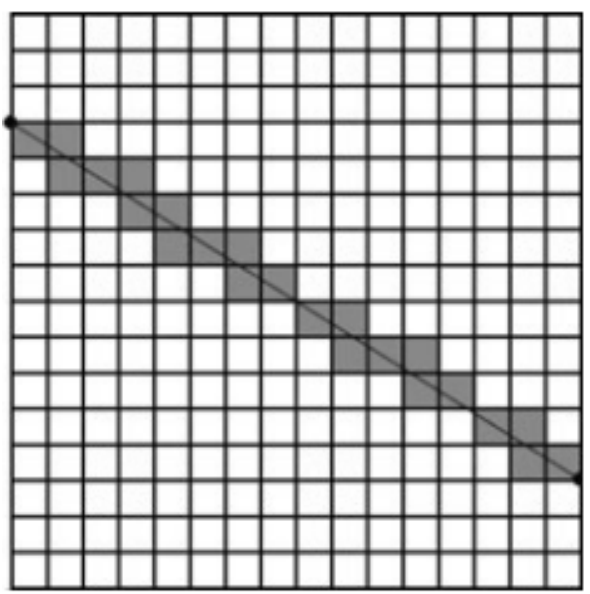

Fig. 3. Beamlet transform as a weighted sum of pixel values along the s headed line 
Where $f_{i_{1}, i_{2}}$ is the gray level value of pixel $\left(i_{1}, i_{2}\right)$ and $\Phi_{i_{1}, i_{2}}\left(x_{1}, x_{2}\right)$ is considered to be the weight function for each pixel. There are a variety of ways to choose $\Phi_{i_{1}, i_{2}}\left(x_{1}, x_{2}\right)$, and in this paper we use average interpolation function.

If $p\left(x_{1}, x_{2}\right)$ represent $\left[i_{1} / n,\left(i_{1}+1\right) / n\right] \times\left[i_{2} / n,\left(i_{2}+1\right) / n\right]$, choose function $\Phi_{i_{1}, i_{2}}$ fulfill the equation:

$$
n^{2} \int_{P\left(x_{1}, x_{2}\right)} \Phi_{i_{1}, i_{2}}\left(x_{1}, x_{2}\right) d x_{1} d x_{2}=\delta_{i_{1}, i_{2}}
$$

\subsection{Algorithm Flow}

According to the characteristics of pavement crack images, the images can be identifying based on Beamlet transform, the basic process is shown in Fig.4.

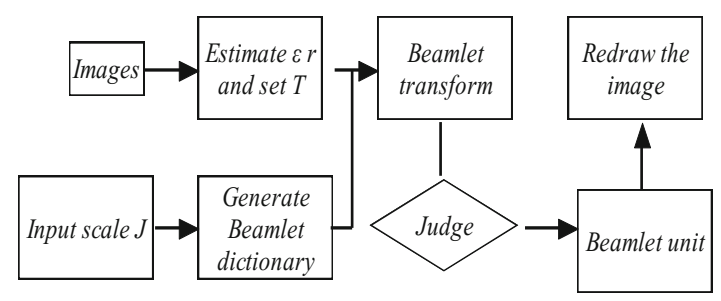

Fig. 4. Algorithm Flowchart

\section{Classification Standard}

The experimental images used in this article were collected on cement road and asphalt road of campus. Under normal circumstances, the pavement crack images have linear characteristic and contain a large number of environmental noise which are not continuous, so that the method based on conventional pixel processing is difficult to detect and classify the crack. Beamlet algorithm has a good robustness because of its line detection, and was suit for crack detection and classification algorithm.

Pavement crack Images during the extraction process can be easily measured by the projection of the crack on the horizontal and vertical directions. Based on the number of branches and the angle of the horizontal direction, the crack can be divided into four types: longitudinal crack, transverse crack, alligator crack and block crack (Table 1). The crack angle is calculated from the start to the end of each crack. If there is a branch exists, the crack deemed as block crack, regardless of the angle of the crack. For each cell block of a crack image, the largest value of Beamlet transform is defined as block cracks length, and the total length of the cracks is the sum of all the blocks along the crack. 
Table 1. The Characteristics of Different Types of Cracks

\begin{tabular}{ccc}
\hline Crack types & Crack angle $\Omega$ & Branch \\
\hline Longitudinal & $\Omega \geq 60^{\circ}$ & no \\
Transverse & $\Omega \leq 30^{\circ}$ & no \\
Alligator & $60^{\circ}>\Omega>30^{\circ}$ & no \\
Block & - & yes \\
\hline
\end{tabular}

\section{$4 \quad$ Experiment and Discuss}

According to the Algorithm flow (fig.4), pavement crack image was processed through image improvement, image enhancement, thresholding, binarization denoising processing and beamlet transform, and the results obtained as shown below. The following figures show the results of the different types of pavement crack image after Beamlet algorithm processing.
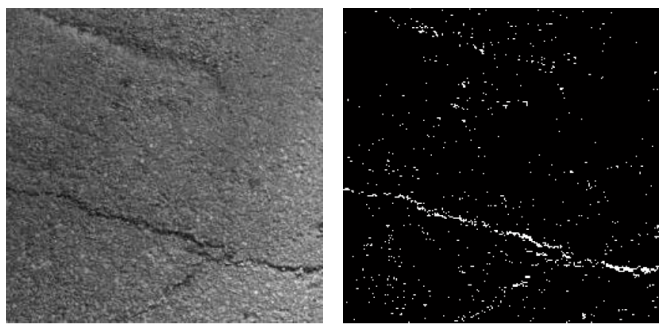

Original image Binary image enhanced and threshold

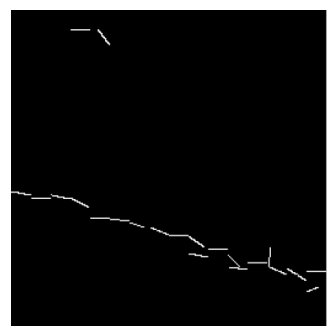

Beamlet transform processing image

Fig. 5. Transverse Crack

Table 2. Transverse Cracks Category

\begin{tabular}{ccccc}
\hline Number & $\begin{array}{c}\text { Crack } \\
\text { angle } \Omega\end{array}$ & Branch & $\begin{array}{c}\text { Crack } \\
\text { length }\end{array}$ & Crack types \\
\hline 1 & $21^{\circ}$ & yes & 18.25 & Transv-erse \\
2 & $6^{\circ}$ & yes & 179.31 & Transv-erse \\
\hline
\end{tabular}


Note that Fig.5 comprises with two transverse cracks, one long and one short. Original crack image was processed through image enhancement, thresholding and beamlet transform, and obtained a good denoising result. The crack information in the original image retained very well, and can be effectively distinction between two types of cracks.
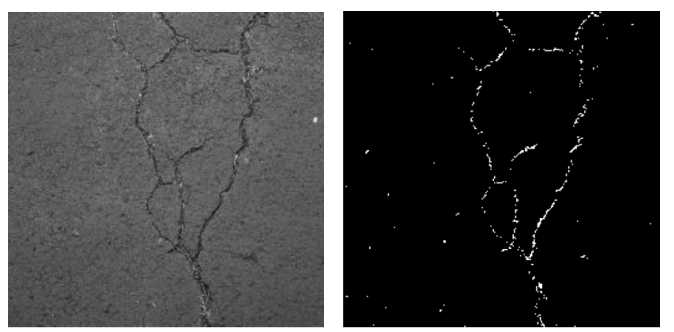

Original image Binary image enhanced and threshold

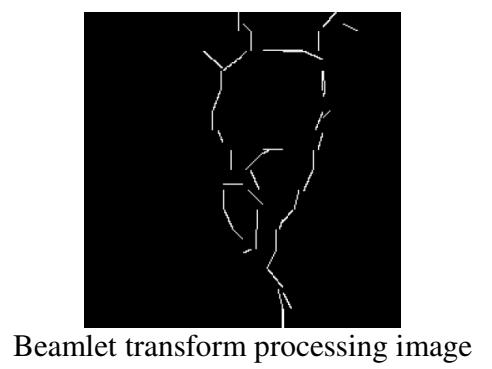

Fig. 6. Alligator

Table 3. Alligator Classification

\begin{tabular}{ccccc}
\hline Number & Crack angle $\Omega$ & Branch & Crack length & Crack types \\
\hline 1 & - & 4 & 448 & Alligator \\
\hline
\end{tabular}

Fig.6 is alligator crack, in which have four branches. According to the presence or absence of the branch, original image can be easily determined as alligator crack. And it can be seen that the method is not susceptible to noise interference, and able to detect the real weak edge.

There was a horizontal crack and a vertical crack in Fig.7. The crack angle of Number 1 is $9^{\circ}$, which is less than $30^{\circ}$, is judged to be transverse cracks. And the crack angle of Number 2 is $67^{\circ}$, which is more than $60^{\circ}$, is judged to be longitudinal cracks. 

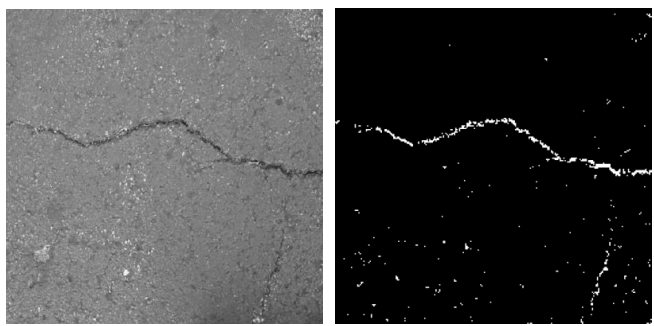

Original image Binary image enhanced and threshold

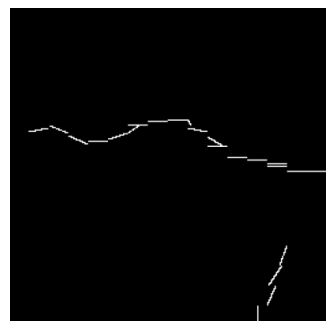

Beamlet transform processing image

Fig. 7. Transverse Crack and Longitudinal Crack

Table 4. Transverse Crack and Longitudinal Crack Classification

\begin{tabular}{ccccc}
\hline Number & Crack angle $\Omega$ & Branch & Crack length & Crack types \\
\hline 1 & $9^{\circ}$ & No & 154.26 & Transve-rse \\
2 & $67^{\circ}$ & No & 53.09 & Longitu-dinal \\
\hline
\end{tabular}
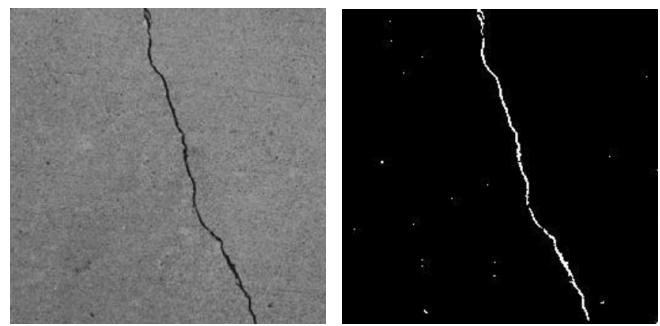

Original image Binary image enhanced and threshold

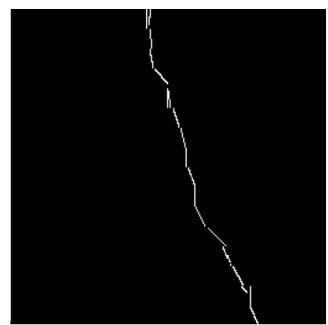

Beamlet transform processing image

Fig. 8. Longitudinal Crack 
Table 5. Longitudinal Crack Classification

\begin{tabular}{ccccc}
\hline Number & $\begin{array}{c}\text { Crack } \\
\text { angle } \Omega\end{array}$ & Branch & $\begin{array}{c}\text { Crack } \\
\text { length }\end{array}$ & $\begin{array}{c}\text { Crack } \\
\text { types }\end{array}$ \\
\hline 1 & $71^{\circ}$ & No & 126.39 & Longitu-dinal \\
\hline
\end{tabular}

Fig. 8 only has one longitudinal crack after image processing. The noise in Original image has removed very well, and obtained a clear crack edge. To test the success rate of the proposed beamlet algorithm, a group of 80 pavement images with different types of cracks is chosen for the experiment. Table 6 presents the statistical results of crack classification. The success rate is calculated by dividing the number of the correctly classified cracks over the total number of cracks chosen. Thus the success rate of the block cracks classification is $90 \%$. From the table it can be seen that the single cracks are easier to classify than the alligator or block cracks.

The results of the experiment show that: Beamlet algorithm can smoothly classify pavement crack images into longitudinal, horizontal, block and Alligator with a good classification results.

Table 6. Success Rate of Crack Classification

\begin{tabular}{cccccc}
\hline Cracks & Number & Longitudinal & Transverse & Alligator & Block \\
\hline Longitudinal & 20 & 20 & & & \\
Transverse & 20 & 1 & 20 & & \\
Alligator & 20 & 2 & 17 & \\
Block & 20 & $100 \%$ & $100 \%$ & $85 \%$ & $95 \%$ \\
\hline Success rate & & & & 1 & \\
\hline
\end{tabular}

\section{Conclusion}

This paper presents a Beamlet transform-based technique to classify the pavement crack images into four types: horizontal, vertical, alligator, and block types. Experiment results demonstrated that the proposed method is very effective with the presence of noise in pavement images. It can be applied on noisy pavement images and classify different types of cracks with a high rate of detection and very low rate of false detection. However, since the Beamlet transform is used to extract linear features, it cannot be used to detect the defects with large area, such as pot holes. In addition more extensive testing is needed to make the algorithm more practical to detect other types of cracks. And the proposed method is capable of finding the length of the cracks but not its width. 


\section{References}

1. Chou, O’Neill, Cheng: Pavement Distress Classification Using Neural Networks. IEEE-94, 0-7803-2129-4/94 (1994)

2. Teomete, E., Amin, V.R., Ceylan, H., Smadi, O.: Digital image processing for pavement distress analyses. In: Proc. of the 2005 Mid-Continent Transportation Research Symposium, Ames, Iowa (August 2005)

3. Bray, J., Verma, B., Li, X., He, W.: A neural network based technique for automatic classification of road

4. Cheng, H.D., Shi, X.J., Glazier, C.: Real-time image thresholding based on sample space reduction and interpolation approach. Journal of Computing and Civil Engineering 17(4), 264-272 (2003)

5. Tsai, Y., Kaul, V., Mersereau, R.M.: Critical assessment of pavement distress segmentation methods. Journal of Transportation Engineering, ASCE 136(1), 11-19 (2010)

6. Ming, Y., Yon, Y., Yuhua, P., et al.: Beamlet transfrom and multiscale linear feature extraction. Acta Electronica Sinica 35(1) (2007) 